\title{
DIFFUSE INTERSTELLAR BANDS - THE UNIDENTIFIED PART OF THE INTERSTELLAR ABSORPTION SPECTRUM
}

\author{
JACEK KREEOWSKI \\ Institute of Astronomy, N. Copernicus University, Toruń, POLAND
}

\begin{abstract}
The unidentified (since 1921) diffuse interstellar bands (DIBs) are discussed together with their relations to other interstellar absorptions such as: continuous extinction, polarization and atomic or molecular absorption lines. It is shown that DIBs do not form the absorption spectrum of one agent, but probably of several (3 or more). DIBs as well as other interstellar absorptions are usually formed in several clouds along a line-of-sight. Thus, they suffer Doppler splitting; the first high resolution profiles free of the latter effect are described. Since single interstellar clouds may differ not only in radial velocities but also in many physical (optical) parameters, the observed interstellar absorptions are ill-defined averages over all clouds situated along any line-of-sight. It is of basic importance to determine not only the single cloud profiles of diffuse bands, but also their relations to other interstellar absorptions in the same clouds. Intensity ratios of DIBs are shown to be sensitive to the shapes of extinction curves, depletion patterns of elements and molecular abundances in the considered clouds. The sensitivity of the DIBs to the variation in polarization is less documented but probably also present. Thus the diffuse lines are presented as the unidentified part of the absorption spectrum of interstellar matter. Their identification depends on the determination of their relations to other interstellar absorptions which must be determined precisely.
\end{abstract}

\section{INTRODUCTION}

This review talk, prepared for presentation at the IAU Symposium, is not a complete review of the results concerning the still unidentified diffuse interstellar bands (DIBs). It is the very biased and personal view of the author, intended to show the observational limitations that anybody attempting to interpret these fascinating spectral features must face sooner or later. As indicated in the title - DIBs are part of the absorption spectrum of the interstellar medium (i.e., ISM). Other members of this "absorption family" are: continuous extinction, polarization, absorption lines of neutral ISM gases and absorption lines of simple molecular species like $C N, C H$ or $C_{2}$. Fig. 1 shows the common situation for all ISM absorptions: being observed in spectra of young $O B$ stars they may be formed in many clouds some of them being remnants of the parent clouds of the observed stars, - others distant and diffuse clouds of neutral hydrogen. It is thus very probable that clouds situated along any line-of-sight may differ in many parameters: density, temperature, velocity, chemical composition, dust content, molecule-content, sizes, internal 


\section{$\mathrm{OB}$ \\ Association}

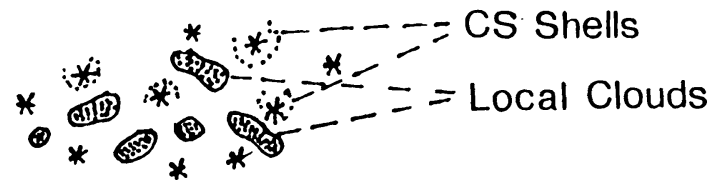

IS

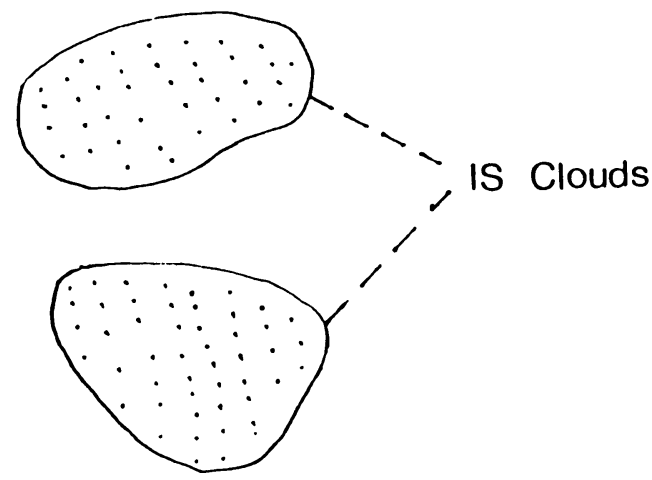

Medium

$$
\uparrow
$$

\section{Observer}

Fig. 1. Interstellar absorptions are usually ill-defined averages over many clouds with possibly very different physical parameters.

structures and shapes of grains, their alignment etc. To understand the processes that are going on in these clouds it is certainly of basic importance to observe the absorption spectra of single clouds. The determination of possible relations between different ISM absorptions may allow us to solve the puzzle of the diffuse bands which remain unidentified since their discovery (Heger, 1921).

The above considerations stress the necessity of extensive observations of bright, nearby, slightly reddened stars - the objects that are most likely to be obscured by only single clouds. It requires very high signal-to-noise $(\mathrm{S} / \mathrm{N})$ spectra to determine DIB profiles because many of them are very shallow, especially those originating in single clouds (Herbig, 1975). The determination of "intrinsic" (single cloud) profiles of the DIBs is possible only when the targets are observed in very high resolution as diffuse bands may suffer Doppler splitting (Herbig and Soderblom, 1982). On the other hand the DIB profiles are usually too wide to clearly show this splitting. Thus in order to properly judge whether the observed profiles are modified or not by the Doppler effect, it is necessary to also observe some sharp lines of interstellar gases (Westerlund and Krełowski, 1988a).

The present day solid state detectors permit one to record spectra with very high $\mathrm{S} / \mathrm{N}$ ratios. This makes it possible to precisely establish intensity ratios of different 
DIBs in different clouds (even if they are very tiny) and thus to decide whether they form one system of absorption features or whether they may be caused by several agents. The identification of DIBs certainly depends on the determination of as many intensity ratios as possible; this task is very difficult as a great majority of DIBs are very shallow features (Krełowski, 1988).

The above mentioned problems may be solved when observing absorptions originating in single clouds or from some considerations of large samples of observational measurements. It seems, however, that individual cases are more clear and safe. It is then necessary to observationally determine the single "bricks" that the ISM is to be built from. The basic observational data that concern the DIBs themselves are presented in $\S 2$; their relations to other ISM absorptions are discussed in $\S 3$. This paper also contains all possible conclusions that may be derived directly from the observations and places some limits to the theoretical speculations on the nature of diffuse bands.

\section{THE KEY OBSERVATIONAL DATA ON THE DIFFUSE SPECTRUM}

The term "diffuse interstellar bands" concerns the approximately 60 spectral features listed by Herbig (1975), Geary (1976), Sanner et al. (1978), and Herbig (1988). They differ significantly in depth, width and shape. It is almost certain that many weak features of this kind still remain undiscovered. Let us consider the problem of relations between different DIBs - certainly one of the basic problems is to determine whether they all originate in the same way from a single interstellar agent or from several materials.

Let's try to compare the intensity ratios of several high quality DIBs, measured by Krelowski and Westerlund (1988) and shown in Figure 2. The comparison strongly suggests different origins of the two well known, relatively strong diffuse bands at $5780 \AA$ and at $5797 \AA$. Fig. 3 gives the comparison of the spectra of the same objects but in the range covering the area of the DIB which has attracted more attention than any other one - the 4430 feature.

It is evident that in $\sigma$ Sco the $4430 \AA$ band is relatively very strong whereas in $\varsigma \mathrm{Oph}$ it is apparently absent (the pretty wide spectral features in this range are stellar lines strongly widened by the fast rotation of the star). Fig. 4 shows the red segments of the spectra of $\zeta$ Per (which resembles $\zeta$ Oph - see Krełowski and Walker 1987) and $\kappa$ Cas. It is clear that the wide 6177 DIB behaves like 4430 whereas $6196,6203,6270$ and the strong, wide 6284 seem to be related to 5780 ; the last two bands: 6376 and 6379 are of very similar intensity in both spectra (of identical reddening) and thus they seem to be related to the strong 5797 DIB.

The above data suggest the division of the DIBs into 3 "families." The question of whether their intensities are related to the abundances of agents or to the excitation conditions can hardly be answered from this material. One more example seems, however, to be worth presentation (Fig. 5). Westerlund and Krełowski (1988c) have observed the star $\gamma$ Ara, assuming it to be a pretty good standard $\left(E_{B-V}=\right.$ 0.05). Surprisingly, some of the DIBs are undoubtedly present in its spectrum (Fig. 5 compares the observed profiles of 5780 and 5797 with the "intrinsic" profiles determined by Westerlund and Krełowski, 1988b).

These observations discovered not only the strong $\mathbf{5 7 8 0}$ band but also a much 


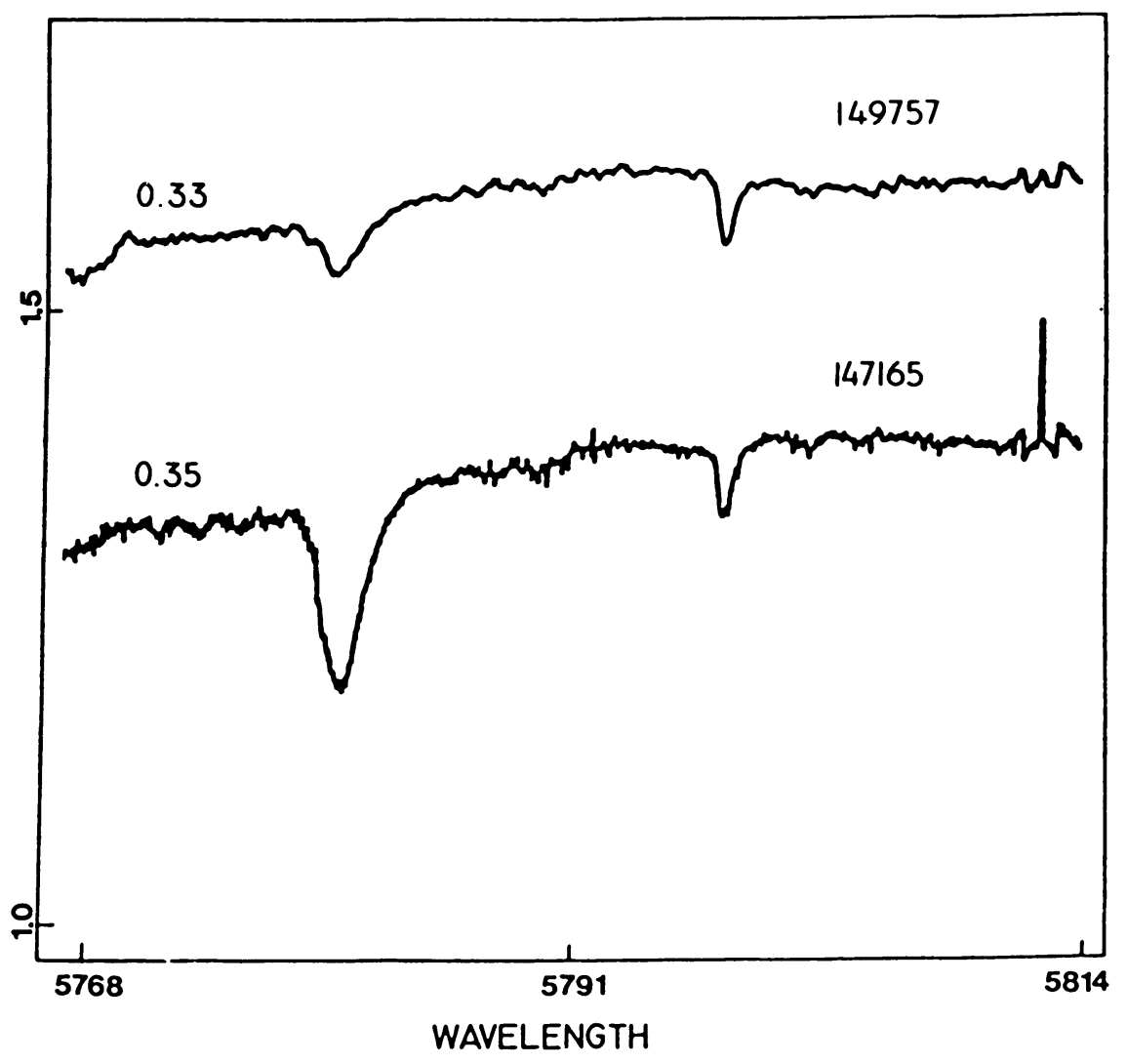

Fig. 2. The 5780 and 5797 diffuse bands observed in the spectra of two nearby stars: 5 Oph (HD149757) and $\sigma$ Sco (HD147165). Note that the intensity ratio varies.

weaker and sharper 6196 component, whereas the normally relatively strong 5797 and 6614 bands (members of another family) are clearly absent. Thus some of the "families" of diffuse bands may not be observed in certain clouds. It is to be emphasized that all the effects of variability of the DIB intensity ratios are strongest and most easy to be presented in single clouds i.e. in the spectra of bright, nearby stars. A similar variability may also be observed in the spectra of distant objects (see e.g. Chlewicki et al., 1987; Josafatsson and Snow, 1987 - Fig. 6). An analysis of such data collections is, however, more difficult: the features observed in every spectrum may originate in several clouds of different optical parameters. This is probably why the above mentioned papers divided the observed diffuse bands into completely different families.

Another difficulty that one can face in dealing with the diffuse band spectrum is that different DIBs sometimes partially overlap. This is clearly shown in Fig. 6. The strong and deep 5780 band is superimposed on the much wider but very shallow 5778. When 5780 is very strong in relation to 5797 , the wide, blue wing appears 


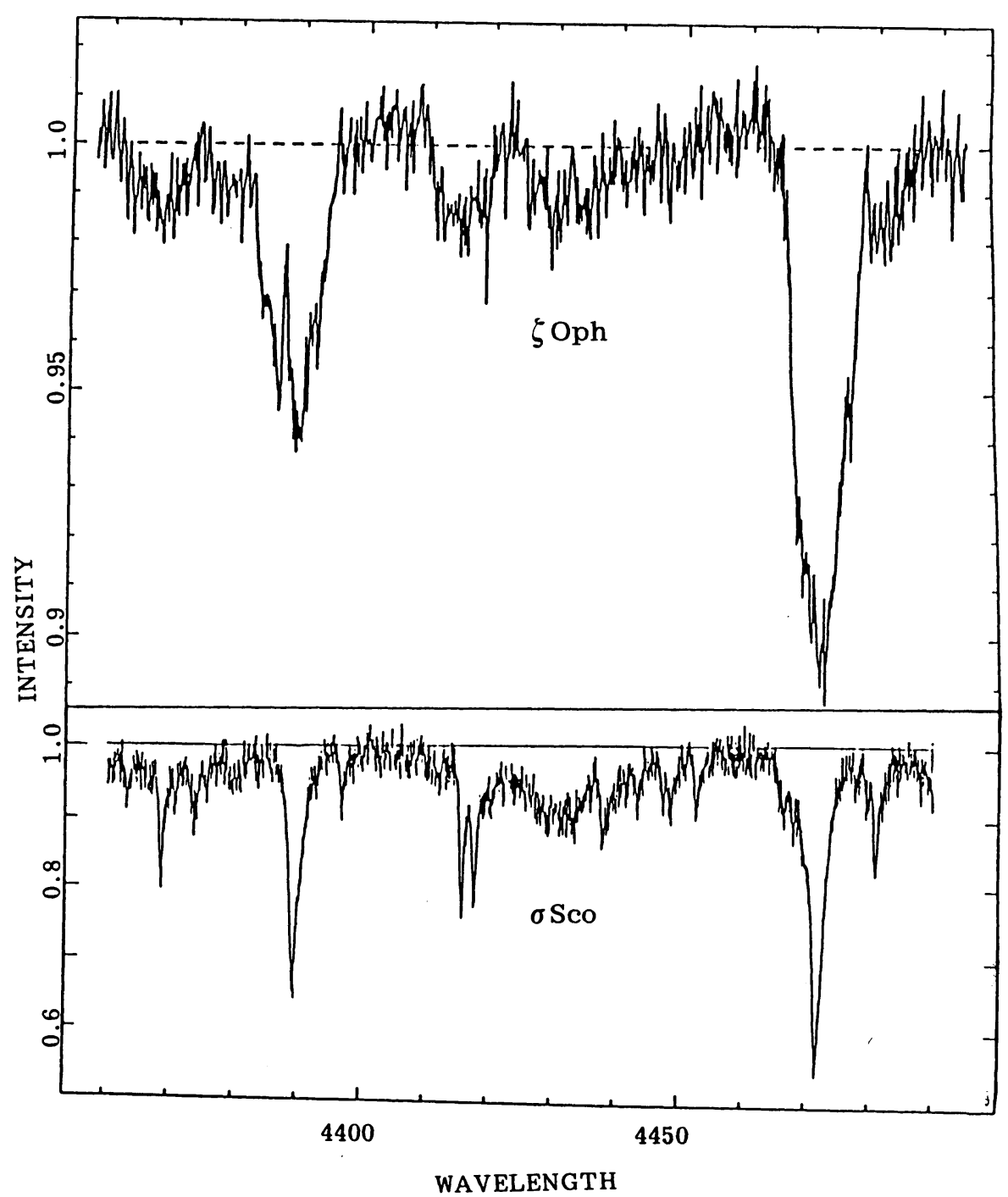

Fig. 3. The 4430 DIB observed in 5 Oph (HD 149757, upper frame) by the author at DAO and $\sigma$ Sco (HD 147165, lower frame) at ESO (courtesy of C. Sterken). The first spectrum apparently lacks the band while in the second its depth reaches $7 \%$ - a value exceptionally high for $E_{B-V}=0.35$. 


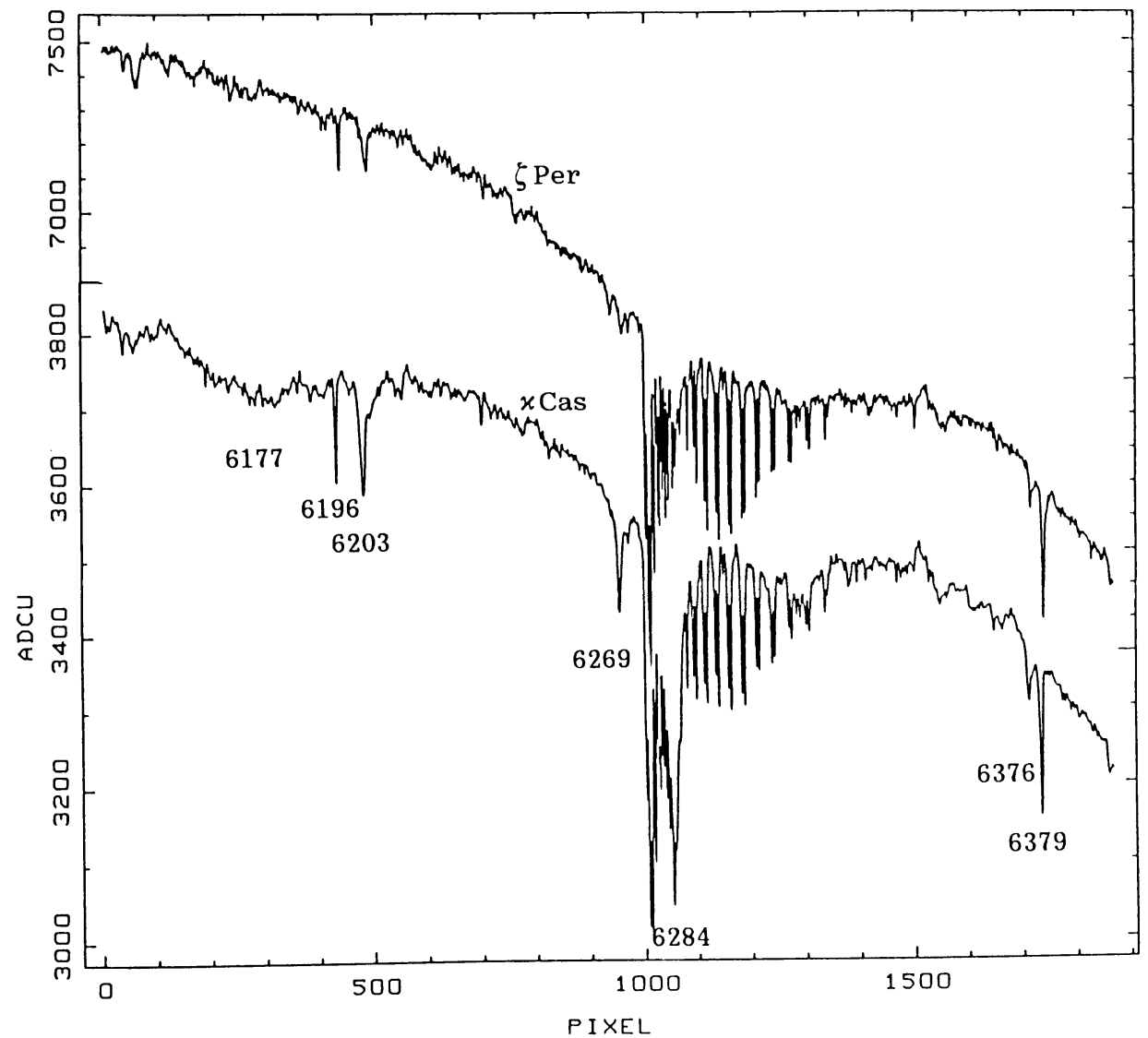

Fig. 4. The red segments of the spectra of $\zeta$ Per (upper) and $\kappa$ Cas (lower) containing several DIBs belonging apparently to 3 different "families."

in the latter. It is certainly the 5795 DIB (Herbig, 1975) - apparently related rather to 5780 than to 5797 - the high resolution but low $\mathrm{S} / \mathrm{N}$ profile of this band is shown in Fig. 5b. The star HD37903, shown in Fig. 6, is the relatively heavily reddened object in which the 5797 DIB (and relatives) is absent. It may be one of the key objects to determine "intrinsic" profiles of the 5780 family not blended with other ones if observed in high resolution and with a high $\mathrm{S} / \mathrm{N}$ ratio.

In order to identify the bands, any comparison of observed DIB profiles with theoretical or laboratory profiles requires the observational determination of profiles not altered by the Doppler effect or by blending with the neighbouring features. A first attempt to complete this observational task was made by Westerlund and Krełowski (1988b). The high resolution observations of DIBs were done together with those of sodium $D$ lines. The latter, being much sharper than any of the diffuse bands, may reveal the presence of Doppler splitting in the observed interstellar absorption spectra and thus can be used to trace the velocity profiles of the differing 

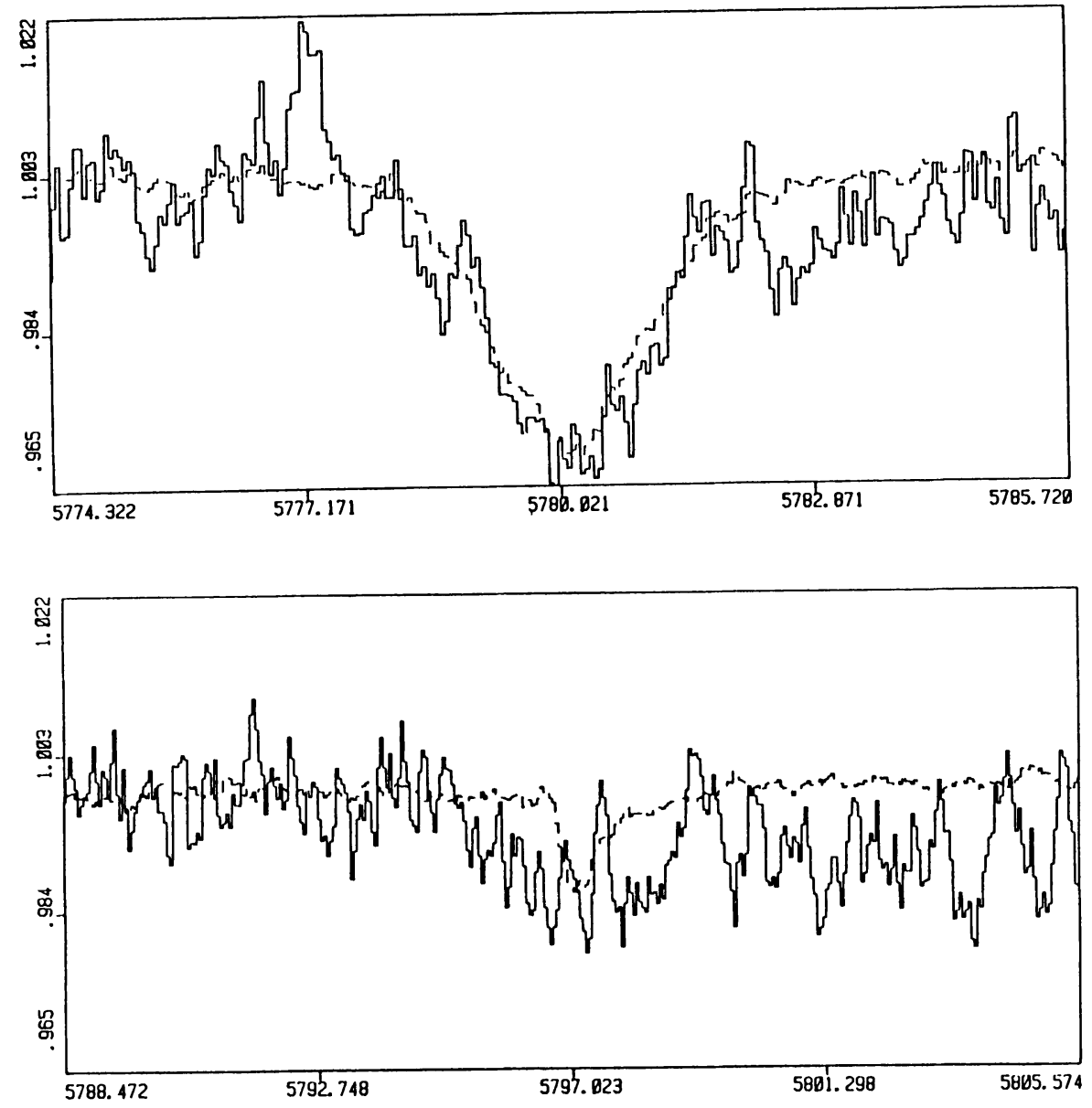

Fig. 5. The 5780 and 5797 DIBs observed in the spectrum of $\gamma$ Ara with the intrinsic profiles superimposed (broken line). The 5797 band is evidently absent. 

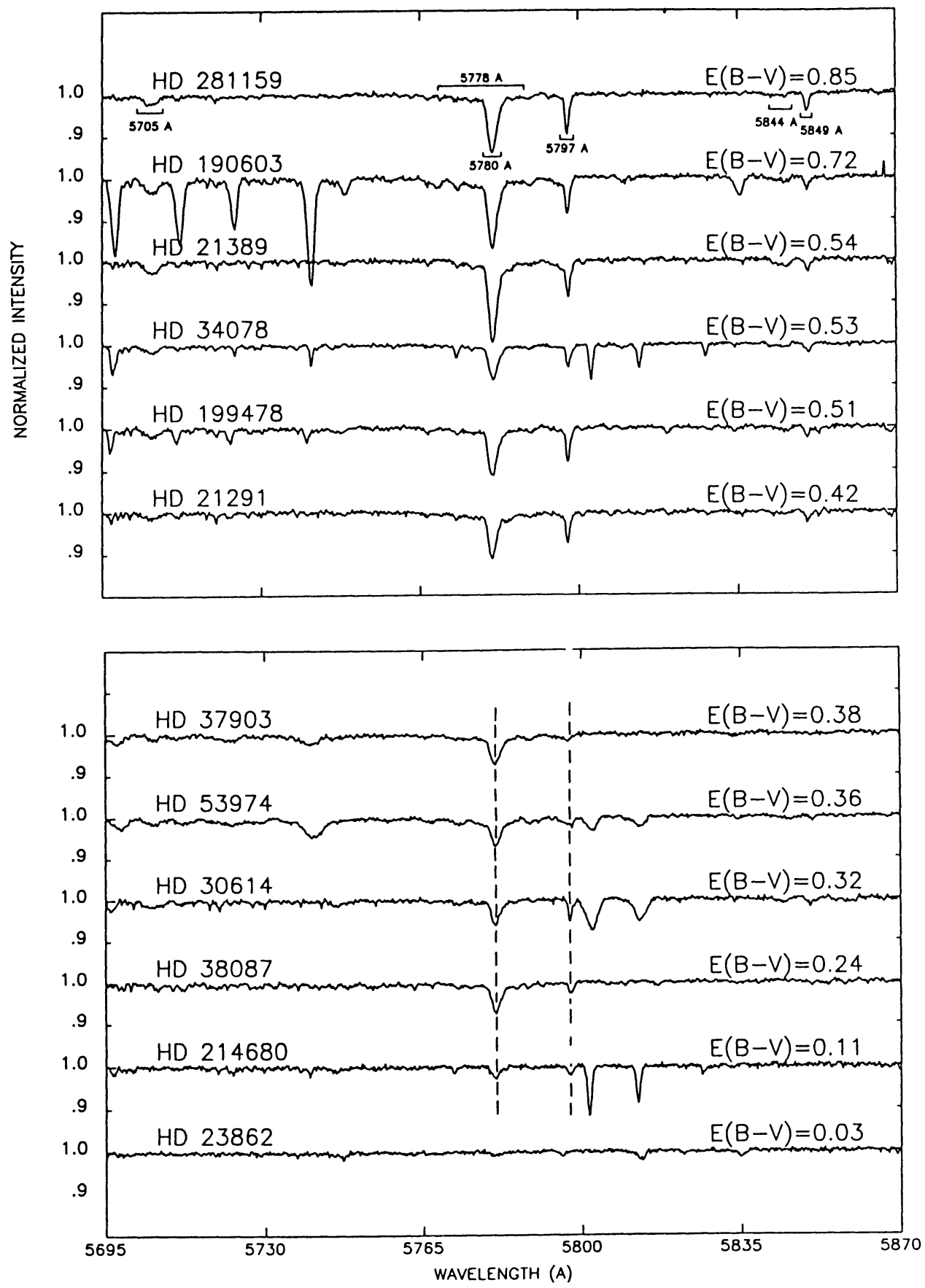

Fig. 6. The survey of yellow DIBs presented by Josafatsson and Snow (1987). Note that 5780 and 5778 overlap as well as 5795 and 5797 - the latter absent in HD37903 (the case similar to $\gamma$ Ara). 


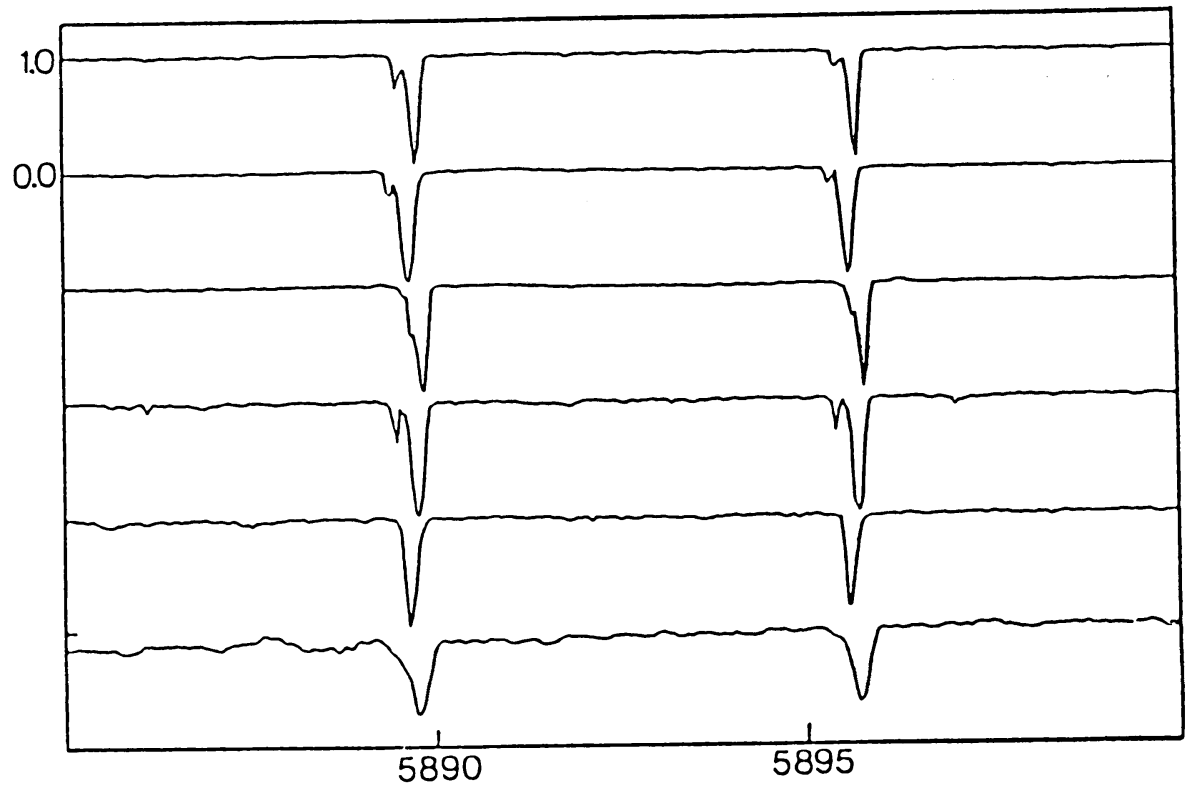

Fig. 7. The profiles of sodium lines observed in the spectra of Sco OB2 stars. From top to bottom the stars are HD: 144217, 149757, 147165, 145502, 142096 and 144470. The latter spectrum has not been used in the DIB analysis of Westerlund and Krełowski (1988b).

contributing clouds (Fig. 7).

This analysis of the Doppler structures allowed us to determine the "intrinsic" (i. e. single cloud) profiles of 5 DIBs: 5780, 5797, 6196, 6284 and 6379. Examples of the 5780 and 5797 are shown in Figs. 8 and 9, respectively. The 5780 and 5797 DIBs differ significantly in their shapes besides being members of different "families" on the basis of their intensity ratios.

The comparison of individual profiles derived from spectra of single stars supplies us with one important conclusion: the shapes (profiles) remain unchanged (not dependent on the parameters that change their intensities). All the considered bands have the same profiles in $\sigma$ Sco and $\zeta$ Oph where their intensity ratios vary rather strongly (Fig. 8 and 9). Also the profiles observed in $\gamma$ Ara (Fig. 5) do not differ from the "intrinsic" ones determined by Westerlund and Krełowski (1988b). Thus the only factor that can change the observed profiles of diffuse bands is the Doppler splitting.

The Doppler effect was discovered in the DIB profiles quite recently (Herbig and Soderblom, 1982), right after high resolution equipment was applied to the DIB observations. However only the sharpest DIBs (like 6196) can be convincingly shown to be composed of two or more Doppler components. Usually we may expect only some modifications of the profiles (width, shape). This fact is well illustrated in Fig. 10 which is taken from the paper of Krełowski and Westerlund (1988). The DIB profiles observed in the spectra of nearby stars, where the obscuration is dominated with single clouds, are identical as mentioned before. On the other hand the profiles 

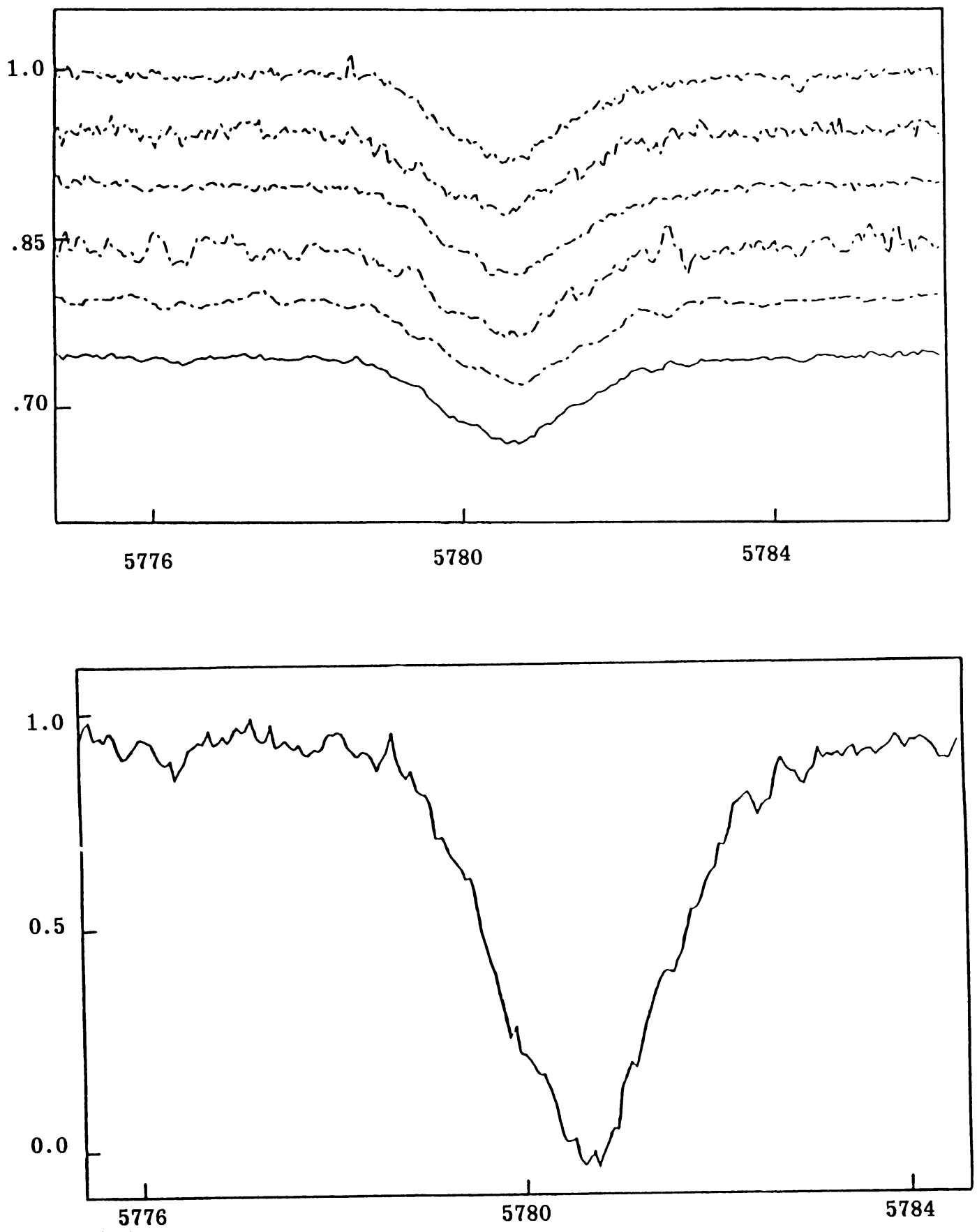

Fig. 8. The individual "intrinsic profiles" of the 5780 DIB derived from the same objects as in Fig. 7. A mean profile (solid line) is also plotted. The mean profile, normalized to the central depth, is shown in the lower frame. 

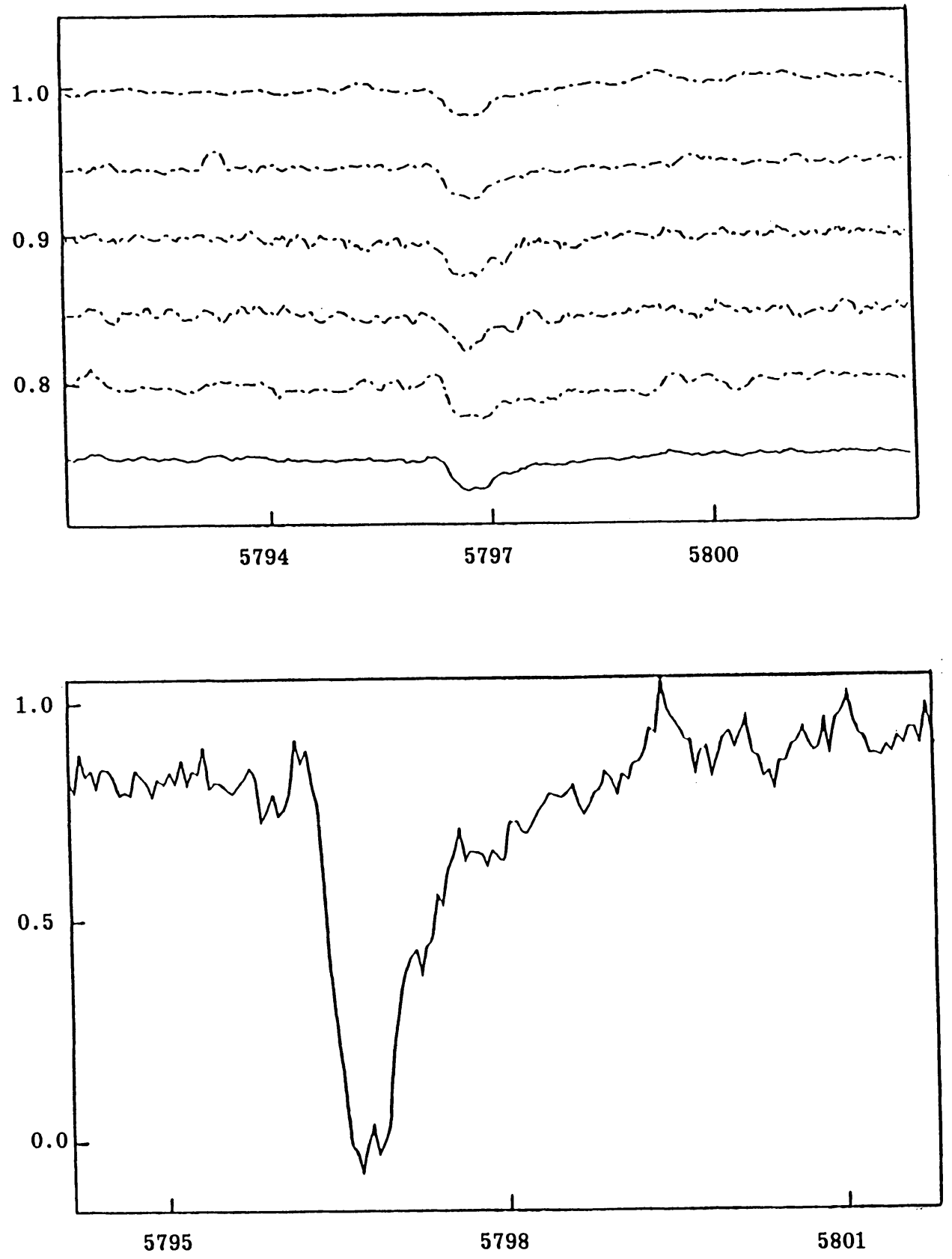

Fig. 9. The same as in Fig. 8 but for the 5797 DIB. Note the similarity of profiles observed in $\varsigma$ Oph and $\sigma$ Sco in both Figures. 
of 5780 and 5797 derived from the spectrum of the distant star HD102997, observed through at least 7 clouds (see the $D$ lines profiles!), are apparently different. The most striking fact is that the intrinsically narrow 5797 DIB is now apparently of a width comparable to that of $\mathbf{5 7 8 0}$. The intensity ratio is, however, somewhere in between $\sigma$ Sco and 5 Oph, suggesting that among the clouds obscuring HD102997 are objects resembling either the first or the second case. The lower frame in Figure 10 proves that the Doppler splitting observed in the sodium lines resembles that seen in the sharp DIBs (6196).

Fig. 10 also gives us another interesting conclusion. The spectrum of the star HD139094 does not contain any of the DIBs in spite of rather substantial reddening. Thus the diffuse band spectrum does not seem to originate in the same dust grains that cause the continuous extinction as was already suggested. Another interesting conclusion may follow the analysis of the HD102997 spectrum. The star is relatively slightly reddened $\left(E_{B-V}=0.42\right)$ and so the optical depth of each of the individual obscuring clouds must be very low. This fact excludes those of the proposed molecular species (as the DIB agents) which require some protection against the diffuse UV radiation.

\section{RELATIONS BETWEEN DIFFUSE BANDS AND OTHER INTERSTEL- LAR ABSORPTIONS}

\subsection{EXTINCTION}

The continuous extinction is the ISM phenomenon usually indicating the presence of some diffuse material between a star and the observer. Only reddened stars have been considered in the previous Section. The results shown above indicate however that one must not expect a tight correlation between the parameters of continuous extinction and intensities of the diffuse bands. On the other hand the same remark that concerns the DIBs is true in the case of the extinction - the DIBs are one of the absorption features which may originate in several clouds along any line-ofsight. Thus it is very interesting to consider the extinction law towards the same targets that have been presented in the previous Section.

Fig. 11 presents the extinction curves derived from the spectra of $\sigma$ Sco and $\$$ Oph using the photometric and UV spectrophotometric data. It is rather evident that these curves differ substantially in their far-UV segments - this effect presented by Bless and Savage (1972) has been confirmed on the base of different data sets by Krelowski and Wegner (1988). This Figure shows that not only the shape of extinction curve, but also the $R$ value (the total-to-selective extinction ratio) varies from star to star (or from cloud to cloud!). Thus the ability of a cloud to generate a certain extinction law is related to that of the formation of at least some of the diffuse bands. This effect may be expected to be veiled when observed in distant stars - only a kind of statistical indication can be derived. In addition, the paper of Krelowski and Wegner shows that the strength and shape of the $2200 \AA$ extinction hump may vary from cloud to cloud. The shapes of DIB profiles remain, however, unchanged as mentioned in the previous Section. Thus we may conclude that the extinction hump is not one of the diffuse bands.

Fig. 12 shows the compilation of recent observational measurements of the $\mathbf{5 7 8 0}$ DIB plotted vs. $E_{B-V}$. It is quite easy to interpret the very large scatter by keeping 

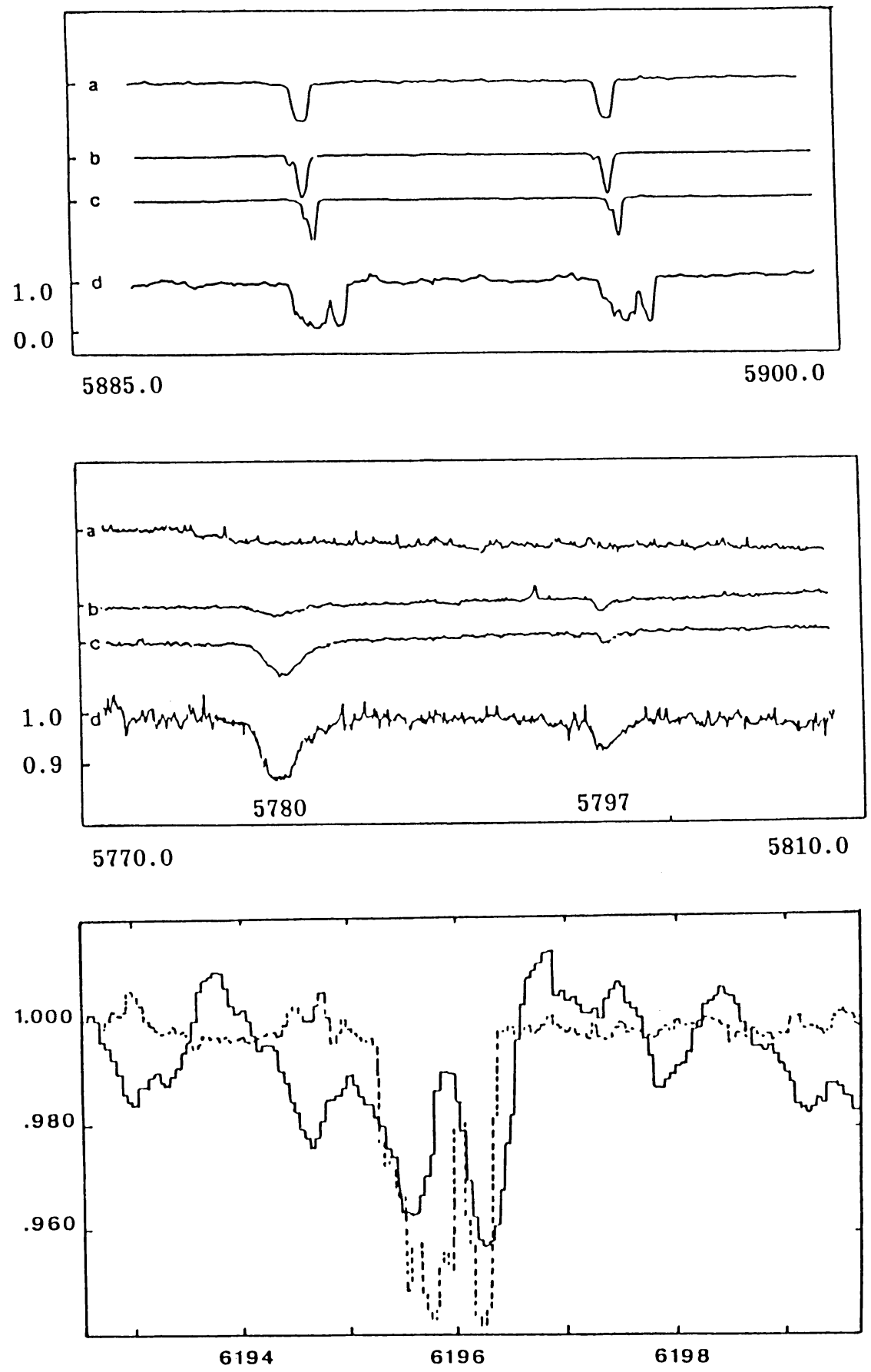

Fig. 10. The sodium lines (top) and 5780 and $5797 \mathrm{DIBs}$ (middle) observed in (from top to bottom) HD: 139094, 149757, 147165 and 102997 (Krelowski and Westerlund, 1988). The profile of the sodium $D_{1}$ line (dashed) is superimposed on that of the sharp 6196 DIB (solid) observed in HD102997 (bottom). 


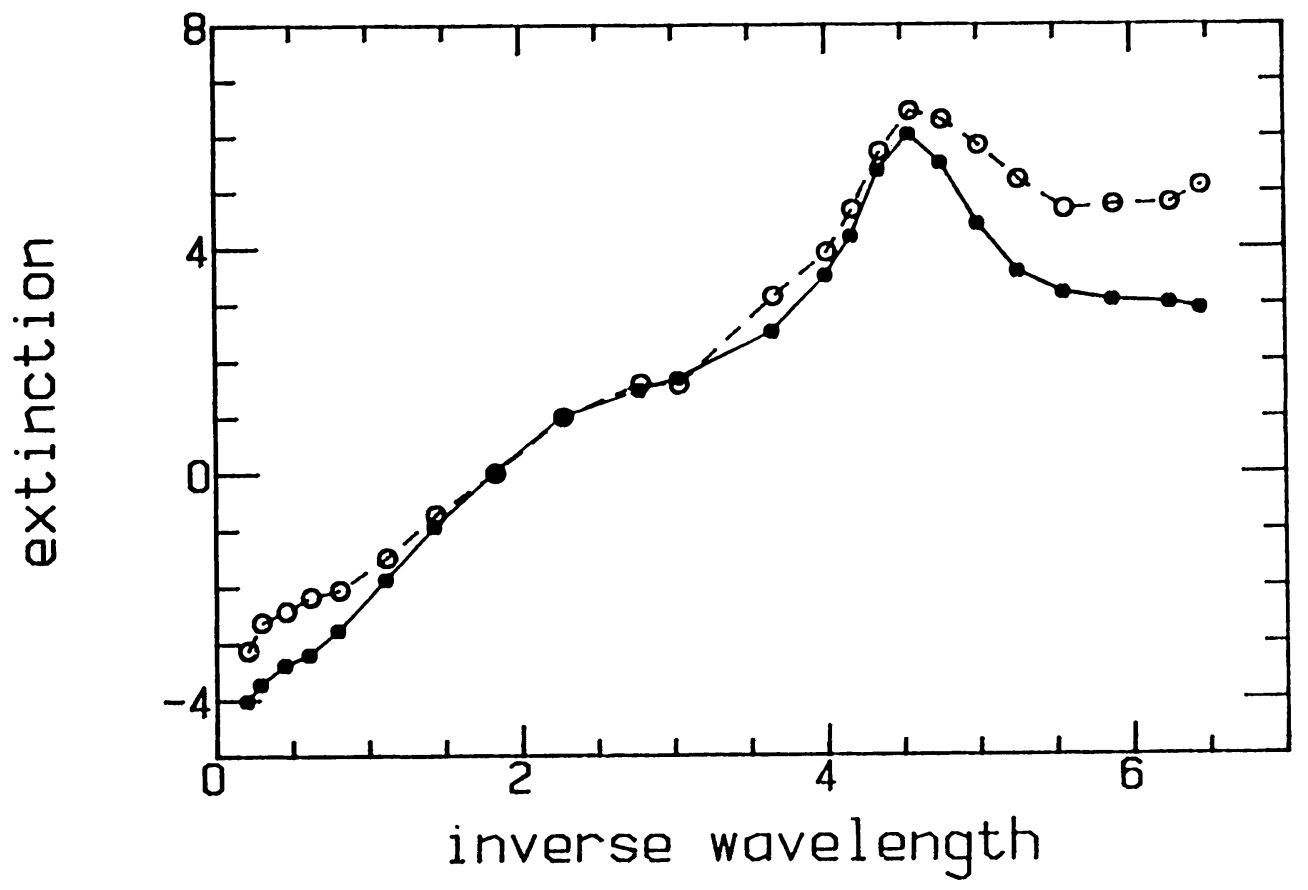

Fig. 11. The extinction curves derived from the spectra of $s$ Oph (open circles) and $\sigma$ Sco (dots) by Krelowski and Wegner (1988). Note the different behavior in IR and far-UV segments of the both curves.

in mind the results shown in the previous Section - DIBs may be completely or partially absent in some of the intervening ISM clouds. Moreover measurements from different sources may differ in cases of overlapping features. The scatter grows substantially towards distant objects and this phenomenon is more difficult to interpret. One important conclusion may be derived from the last Figure: the low-tomoderately reddened stars clearly suggest that DIBs vanish completely when $E_{B-V}$ goes to zero. Thus DIBs are rather unlikely to be present in some unreddened spectra as suggested by extrapolating the linear fits to the data of Fig. 12 including the heavily reddened stars. There is, however, an additional interesting question: why does the slope of the relation found by Chlewicki et al. (1986) for the 5797 DIB differ by a factor of 3 from that suggested by the data of Herbig (1975)? Possibly it is due to some regional variations of the properties of interstellar matter. Possibly this is why different conclusions can be derived from a statistical treatment of stellar samples if only very crude parameters of the ISM (equivalent widths of DIBs and $\left.E_{B-V}\right)$ are taken into account.

Let's consider the survey of the 4430 DIB done by Krełowski et al. (1987). This survey, based on a homogeneous set of high quality data, concerns mostly bright stars that have also been observed with the ANS satellite. The upper frame in Fig. 13 shows the plot of the far-UV colour excess (between the bands 1550 and $1800 \AA)$ vs. $E_{B-V}$. It indicates how the stars were divided into groups obscured by 


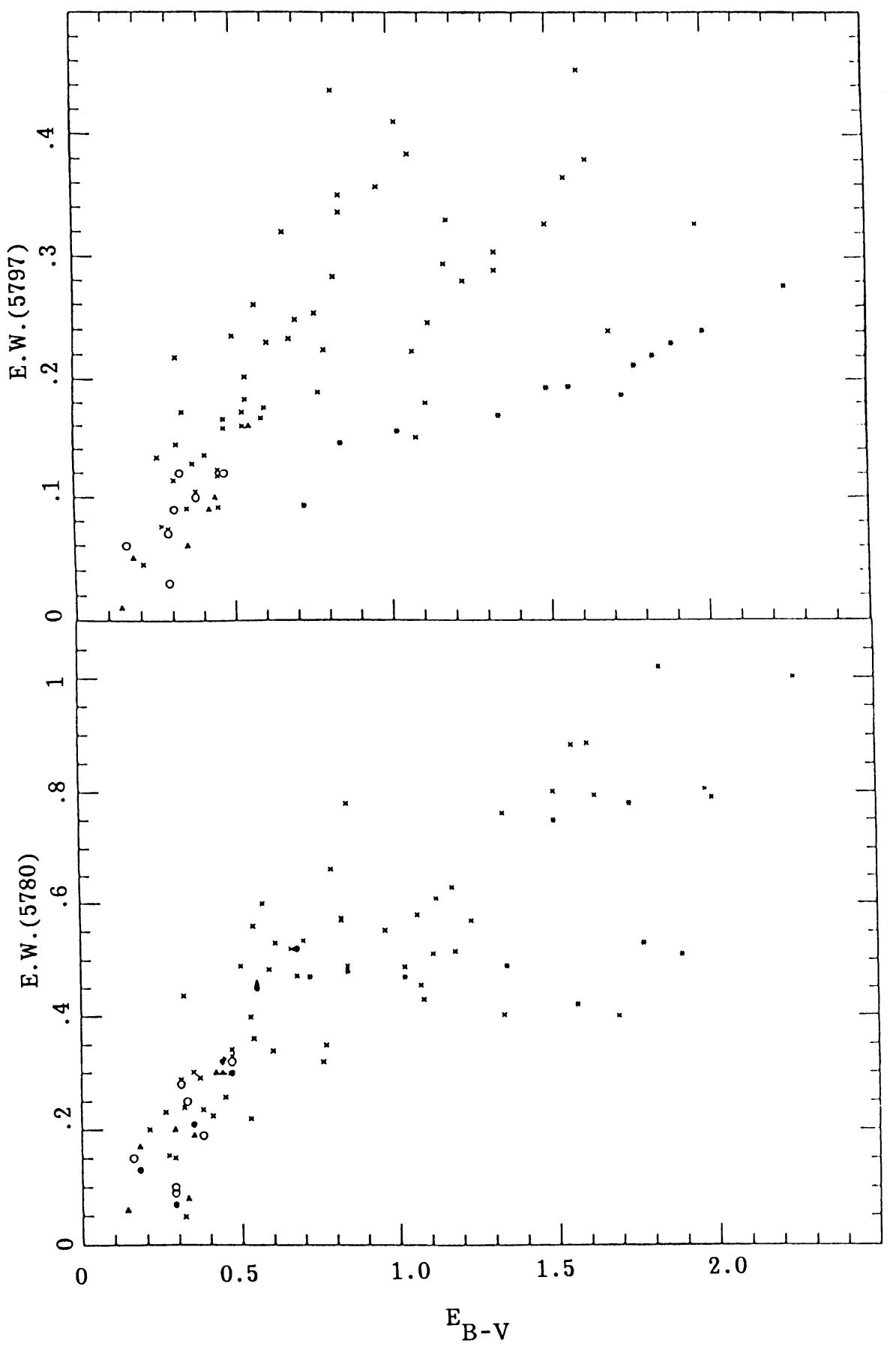

Fig. 12. The equivalent widths of 5797 (upper frame) and 5780 (lower frame) DIBs plotted vs. $E_{B-V}$; crosses - Herbig (1975), triangles - Danks and Lambert (1976), dots - Snell and Vanden Bout (1981), asterisks - Chlewicki et al. (1986), open circles - Krełowski and Walker (1987). 
the clouds of high and low (relatively) far-UV extinction. The factor that changes the shape of the extinction curve in the far-UV has apparently no influence on the segment from 3300 to $2200 \AA$ ( Fig. 13, middle frame). The lower frame in Fig. 13 proves that this factor influences the central depth of the $4430 \AA$ band: it is usually weaker when the far-UV extinction is strong in accordance with the suggestion of Figs. 10 and 11. Thus considerations of individual cases as well as statistics lead to the same conclusion: intensities of certain diffuse bands (in relation to $\left.E_{B-V}\right)$ decrease in the presence of strong far-UV extinction. The effect is, however, evidently stronger when individual cases are being considered.

Finally we may conclude: a colour excess does not characterize fully the extinction curve. The shape of the curve (especially when derived from a single cloud) may reveal, however, the ability of the interstellar material to produce some of the diffuse bands. It has been shown recently (Krełowski and Wegner, 1988) that some of the ISM extinction curves originating in single clouds differ considerably from those of $\sigma$ Sco or $\varsigma$ Oph but the behaviour of DIBs in these cases is less clear. It is only suggested that the very peculiar extinction law in $\phi$ Per is observed together with the exceptional weakness of 5780 and 5797 DIBs.

\subsection{POLARIZATION}

To analyse the possible relations of polarization to other interstellar absorption characteristics is especially difficult. The reason is that the additional factor we have to deal with is the grain alignment which, changing from place to place, can reduce the polarization by dilution when the beam crosses the next cloud. This is the exclusive property of the polarization - all other ISM absorptions get stronger after the beam crosses an additional cloud. Thus the analysis of polarization in relation to diffuse bands makes sense only in cases of single clouds.

The polarization curves of $\sigma$ Sco, $\zeta$ Oph and $\phi$ Per are shown in the Fig. 14 . The differences are quite evident suggesting that an analysis of polarization may be a useful diagnostic tool to derive some conclusions concerning DIBs (Papaj and Krelowski, 1988). The curves differ in the wavelengths of maximum polarization and in shape, confirming that the clouds obscuring these stars differ seriously not only in physical parameters but also in grain sizes, shapes and alignments. The polarization curve (as well as extinction) of $\phi$ Per differs substantially from the others but our information concerning the DIBs to this star are rather scarce. On the other hand, the latter polarization curve has a maximum in the ultraviolet; thus the value of the total-to-selective extinction ratio does not seem to be proportional to the $\lambda_{\max }$ of polarization - the $R$ value reaches $\approx 15$ for $\phi$ Per.

\subsection{ATOMIC AND MOLECULAR LINES}

As shown in $\S 2$ the high resolution profiles of the lines of interstellar sodium may reveal the structure of the ISM towards the observed stars. The upper and lower frame in Fig. 10 shows the similarity of Doppler splitting in a DIB and in $D_{1}$ line profiles. It is to be emphasized, however, that DIB intensities are not obviously related to those of sodium lines or any other atomic lines. The intensity differences between the sodium lines depicted in the upper frame of Fig. 10 are related neither to differences in reddening nor to variations in the DIB intensities. 


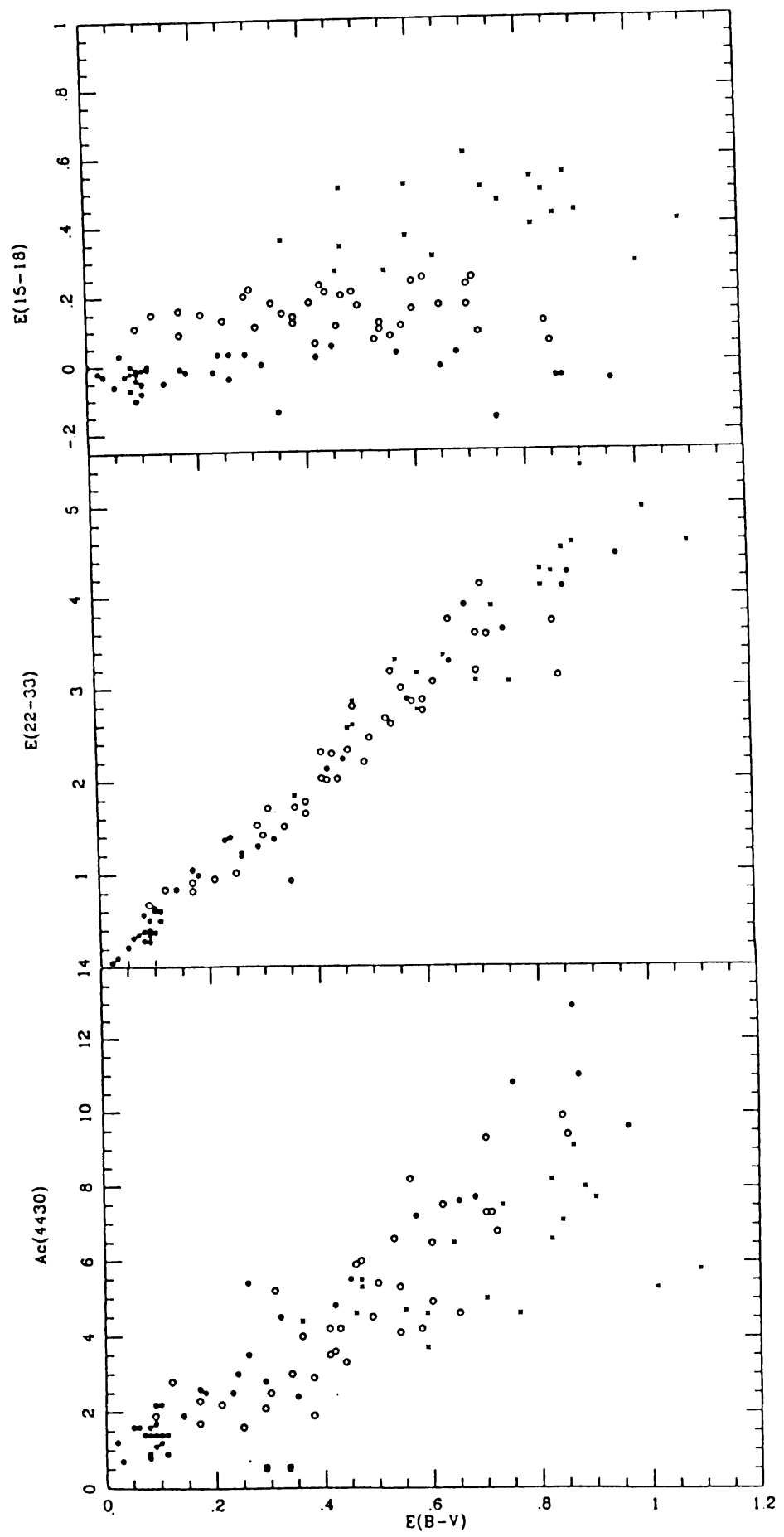

Fig. 13. The $1550-1800 \AA$ (top) and $2200-3300 \AA$ (middle) ANS color excesses of the stars surveyed by Krełowski et al. (1987); the central depths of the $4430 \mathrm{DIB}$ are depicted below. 


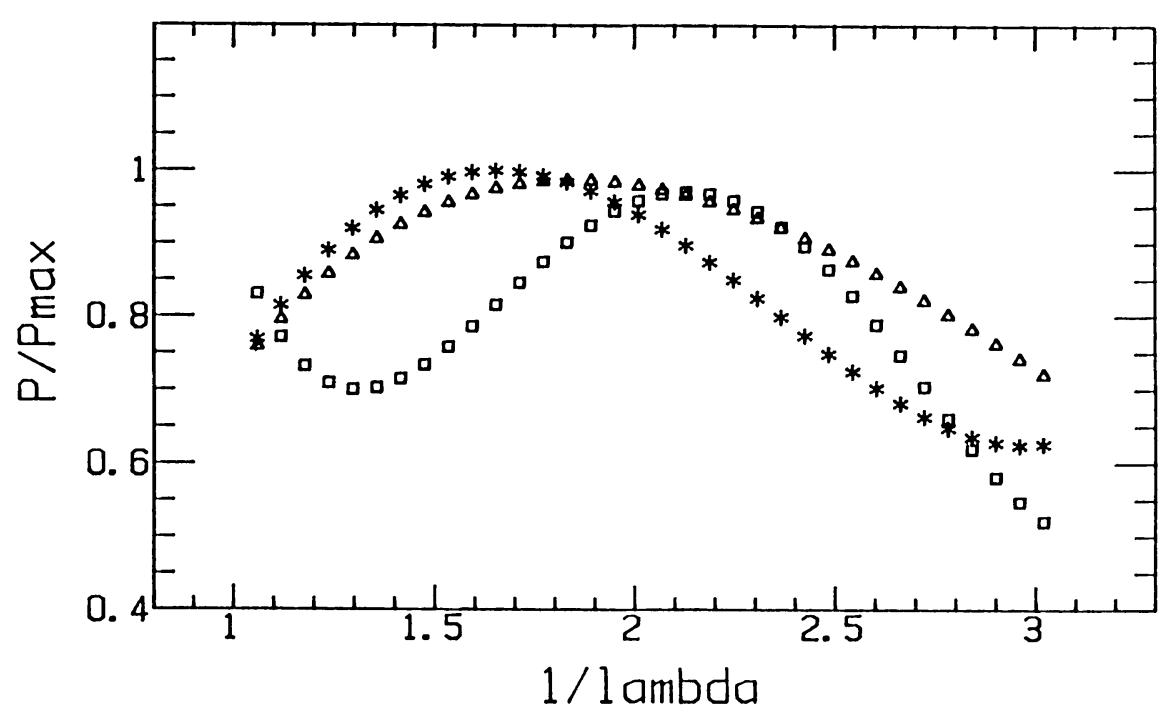

Fig. 14. The polarization curves characteristic for $\sigma$ Sco (triangles), $\varsigma$ Oph (asterisks) and $\phi$ Per (squares). Note the varying shape and the position of $\lambda_{\max }$.

It is very interesting to check if the abilities of single clouds to produce DIBs are related to the depletion patterns in the same clouds. The excellent set of profiles of ISM atomic lines determined with the aid of the Copernicus spectrometer (Bohlin et al., 1982) has been used to investigate the objects producing similar diffuse spectra e.g. 5 Oph and $\sigma$ Sco plus the members of their "families" (Niedzielski and Krełowski, 1988). The equivalent widths (and thus the column densities) of several elements that may be expected to be depleted in the ISM are evidently weaker in relation to $E_{B-V}$ in the $\zeta$ family than in the $\sigma$ one $(M n, M g, F e)$. The column densities of other elements e.g., $\mathrm{Cl}$, are very similar in both cases, however in the $\sigma$ family, ionized chlorine (absent in the $\zeta$ one) is evidently observed. On the other hand some of the atomic lines may be relatively stronger in the clouds producing weak 5780 or 4430 DIBs. The $K I \lambda 7699$ absorption line is clearly stronger in the spectra of $\varsigma$ Oph or $\varsigma$ Per than e.g. in $\sigma$ Sco (Hobbs, 1974).

Let's mention another important observational fact: absorption lines of simple molecular species observed in the spectra containing diffuse features of different intensity ratios show evidently different (in relation to $E_{B-V}$ ) column densities (Danks et al., 1984; Federman et al., 1984). The absorption lines of simple molecular species $\left(C N, C H, C_{2}\right)$ are very strong in the spectra of such stars like $\zeta$ Per or $\zeta$ Oph - almost an order of magnitude stronger than in e.g. $\sigma$ Sco. These molecules may be (together with the dust grains) the "sink" for the atoms that seem to be so underabundant in the same clouds. 
The data presented in this Section suggest strongly that several different kinds of ISM clouds really exist in space. They may differ in extinction law, in degree and positional angle of polarization, in depletion patterns, in abundance of molecules and, of course, in their abilities to produce diffuse interstellar bands. It is to be mentioned, however, that the proposed single clouds do not exhaust all possibilities. Combinations of the extinction curves presented by Krelowski and Wegner (1988) do not allow one to construct the extinction curve for a distant object such as the Per OB1 association (Krełowski and Strobel, 1987) - the latter is steeper in the far-UV even than that of $\zeta$ Oph. Thus the proposed curves concern probably nearby, local clouds - not a truly interstellar diffuse component. The ability of this component to produce DIBs as well as other ISM absorption features remains unknown.

\section{CONCLUSIONS}

The data discussed above lead to several important conclusions concerning the nature of interstellar dark matter. Probably the most important one is that the ISM consists of several kinds of clouds - differing rather significantly in their physical parameters. It is hard to decide how many kinds of different clouds really exist in interstellar space but it is certain that we cannot expect a "continuum" of physical parameters among the dark clouds. The observed HI clouds differ usually simultaneously in extinction laws, elemental depletion patterns, molecular abundances (in relation to a colour excess) and the abilities to produce the diffuse interstellar bands. The existence of these different clouds allows to derive several conclusions concerning the heroes of this paper:

1. DIBs form several "families," defined as systems of these features among which the intensity ratios remain unchanged from one cloud to another.

2. Profiles of diffuse bands originating in clouds of different properties always maintain the same shape; they differ only in intensities.

3. Some interstellar clouds do not produce DIBs at all; some others produce only certain "families."

4. The relationships with $E_{B-V}$ indicate that diffuse bands do not exist in the absence of reddening.

5. DIBs originate in very tiny clouds (e.g. $\gamma$ Ara); thus their agents seem to be resistant against the diffuse UV radiation.

6. Relative intensities of the diffuse bands depend on the shape of the extinction law: the 4430 and 5780 "families" are evidently attenuated when the far-UV extinction is enhanced in relation to $E_{B-V}$; the $2200 \AA$ extinction hump seems not to belong to any of the DIB "families."

7. The situation of enhanced far-UV extinction seems to be accompanied by relatively high column densities of simple molecular species (enhanced by a factor $\approx 7$ ); no information about more complex molecules is presently available.

8. The relative intensities of the diffuse bands are related in quite a complicated way to the depletion of many elements inside the clouds considered. 
9. A relation between the ability of a cloud ability to produce DIBs and to polarize the starlight evidently exists but is still far from being properly established; the polarization curves clearly change when the diffuse spectrum changes.

10. The lack of diffuse bands in some ISM clouds as well as in all observed CS shells (Snow and Wallerstein, 1972) proves they do not originate in the same dust grains that produce the continuous extinction.

The conclusions listed above tell us which materials are not the agents of diffuse bands; they don't tell us, however, which are. Some of the conclusions are only suggestive - thus it would be very valuable to check whether these suggestions are correct or not. It is to be emphasized once again that observations of nearby stars, obscured by single clouds are of basic importance for the understanding of the physical processes inside the interstellar medium. The present paper does not include any considerations based on IR or radio data which may also be of great importance. So the diffuse bands remain to be a fascinating puzzle but it seems that in the eighties we have made an evident step forward to solve it.

ACKNOWLEDGEMENTS. This research was partly supported financially by the project RPBR-RR-I.11-1.

\section{REFERENCES}

Bless, R. C., Savage, B. D. 1972, Ap. J., 171, 293.

Bohlin, R. C, Hill, J. K., Jenkins, E. B., Savage, B. D., Snow, T. P., Spitzer, L., York, D. G. 1982, Wisconsin Astrophysics, 156.

Chlewicki, G., van der Zwet, G. P., van IJzendorn, L. J., Greenberg, J. M. 1986, Ap. J., $305,455$.

Chlewicki, G., de Groot, M. S., van der Zwet, G. P., Greenberg, J. M., Alvarez, P. P., Mampaso, A. 1987, Astr. Ap., $178,131$.

Danks, A. C., Federman, S. R., Lambert, D. L. 1984, Astr. Ap., 180, 62.

Danks, A. C., Lambert, D. L. 1976, M. N. R. A. S., 174, 571.

Federman, S. R., Danks, A. C., Lambert, D. L. 1984, Ap. J., 287, 219.

Geary, J. C. 1976, in IAU Colloquium 40, Astronomical Applications of Image Detectors with Linear Response, ed. M. Duchesne and G. Lelievre (Meudon: Obs. de Paris), p. 28-1.

Heger, M. L. 1921, Lick Obs. Bull., 10, 146.

Herbig, G. H. 1975, Ap. J., 196, 129. $1988, A p . J$.(in press).

Herbig, G. H., Soderblom, D. R. 1982, Ap. J., 252, 610.

Josafatsson, K., Snow, T. P. 1987, Ap. J., \$19, 436.

Krełowski, J. 1988, Pub. A. S. P., (in press).

Krełowski J., Strobel, A. 1987 Astr. Ap., 175, 186.

Krełowski, J., Wegner, W. 1988, Astron. Nachr. (in press).

Krełowski, J., Westerlund B. E. 1988, Astr. Ap., 190, 339.

Krełowski, J., Walker, G. A. H. 1987, Ap. J., 312, 860.

Krełowski, J., Walker, G. A. H., Grieve, G. R., Hill, G. 1987, Ap. J., 316, 449.

Niedzielski, A., Krełowski, J. 1988 Astr. Ap.(submitted).

Papaj, J., Krełowski, J. 1988 Astr. Ap.(submitted).

Sanner, F., Snell, R. L., Vanden Bout, P. A. 1978, Ap. J., 226, 460.

Snell, R. L., Vanden Bout, P. A. 1981, Ap. J., $244,844$.

Snow, T. P., Wallerstein, G. 1972, Pub. A. S.'P., 84, 492.

Westerlund, B. E., Krełowski, J. 1988a, Astr. Ap., 189, 221.

. $1988 b$, Astr. Ap.(in press).

- 1988 c, Astr. Ap.(in press). 